\title{
Probing the Strain States in Nanopatterned Strained SOI
}

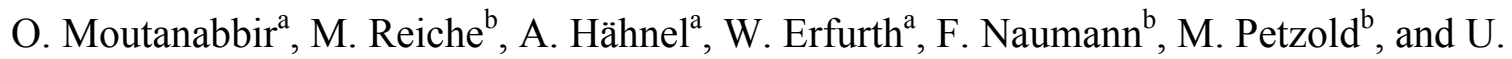 \\ Gösele $^{\mathrm{a}}$ \\ ${ }^{a}$ Max Planck Institute of Microstructure Physics, Weinberg 2, D 06120 Halle (Saale), \\ Germany \\ ${ }^{b}$ Fraunhofer for Mechanics of Materials, Walter-Hülse-Strasse 1, D 06120 Halle (Saale), \\ Germany
}

\begin{abstract}
Using strained SOI as a strategy to enhance the performance of Sibased electronic devices raises fundamental questions about the stability of the strain during different processing steps. In this work, we elucidate the influence of nanoscale pattering, a crucial step in device fabrication, on the strain states. UV micro-Raman and high resolution transmission electron microscopy were employed to quantify the strain in the strained layers. Post-patterning strain in different nanostructures was evaluated by UV micro-Raman. Our data demonstrate that the formation of free surfaces upon pattering leads to a partial relaxation of the strain. The extent of the relaxation was found to depend on the lateral dimension and the geometry. A detailed mechanistic picture is presented based on 3D finite element simulations.
\end{abstract}

\section{Introduction}

Strain engineering is regarded as a key factor to respond to the relentless demand of higher performance CMOS devices. Recent studies showed an increase in the carrier mobility for devices fabricated on strained silicon layers (1-3). Models suggest that a strained Si lattice can give hole mobilities of up to 4 times the unstrained value, and electron mobilities up to 1.8 times the unstrained value (4). Uniaxial or biaxial strain are the two presently applied ways for the manipulation of the carrier mobility in the channels of Si field effect transistors [see Ref. 5 and references therein]. Each type of strain influences the performance in a different way, because each has a different effect on Si band structure. Both uniaxial and biaxial strain break the 12-fold symmetry of the unstrained Si band structure (6). For biaxially strained Si, the mobility enhancement originates from scattering reduction. Further improvement of device performance can be achieved by combining the benefits of strained layers with the advantages of silicon-oninsulator (SOI) in the same substrate of strained Si thin layer directly on insulator commonly known as strained silicon-on-insulator (SSOI) (7).

The introduction of SSOI in device fabrication raises fundamental questions about the evolution and stability of the strain during different processing steps. It was demonstrated that the strain in SSOI can be maintained during high temperature annealing or processing (8). However, the shrinkage of the dimensions during the sub-micro- and nanoscale pattering of strained thin films were found to affect the amount and distribution of the strain $(9,10)$. In a recent report, we showed that the relaxation of the biaxial strain upon nanostructuring of SSOI is size-dependent (10). Further insights into the relaxation 
are presented in this paper. Understanding this phenomenon is necessary to develop a quantitative and predictive model of the performance of SSOI-based devices. Moreover, deciphering the behavior of the strain during nanopattering process is crucial for the optimization of the fabrication of the SSOI wafer per se.

\section{Fabrication of SSOI and characterization strain}

8-inch SSOI wafers were used in this study. The strain was generated by the heteroepitaxial growth of Si thin film on $\sim 500 \mathrm{~nm}$-thick $\mathrm{Si}_{1-\mathrm{x}} \mathrm{Ge}_{\mathrm{x}}$ buffer layer, which was relaxed by helium implantation and thermal annealing (11). The tensile strained thin layer was then transferred to a $\mathrm{SiO}_{2} / \mathrm{Si}$ handle wafer by combining wafer bonding, hydrogen ion-induced thin layer transfer, and etch-back methods $(5,12)$. The amount of the strain is controlled by adjusting the content of Ge in SiGe buffer layer. The initial thickness of the strained layer is in the range of 15-30 nm. Thicker layers were obtained by an additional homoepitaxy. Figure 1 displays a cross-sectional transmission electron microscopy (XTEM) image of a $15 \mathrm{~nm}$-thick SSOI substrate. It is noticeable that the strained film has a uniform thickness and it is defect-free as demonstrated by the high resolution image and the electron diffraction pattern.
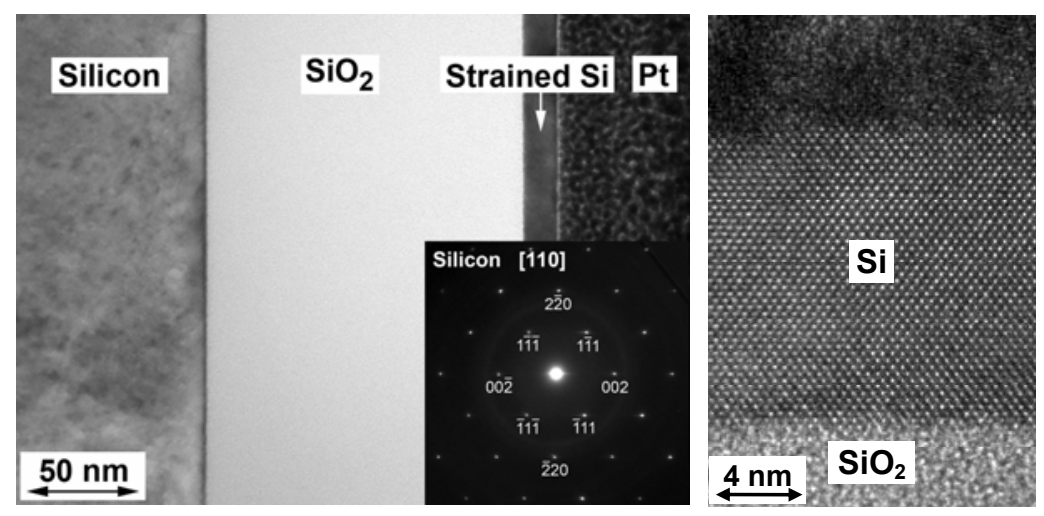

Figure 1. XTEM (left) and HRXTEM (right) images of a $15 \mathrm{~nm}$-thick SSOI substrate. Inset: Electron diffraction pattern.

UV micro-Raman is used to characterize the biaxial strain. The measurements were performed in backscattering geometry by a LabRam HR800 UV spectrometer with a 325 $\mathrm{nm} \mathrm{He}-\mathrm{Cd}$ laser line corresponding to a penetration depth of $\sim 10 \mathrm{~nm}$ in $\mathrm{Si}$. A $40 \times$ objective was used to focus the laser beam to a spot of $\sim 800 \mathrm{~nm}$ in diameter at the surface of the sample. To avoid local heating effects, the laser power was kept below $2 \mathrm{~mW}$. The backscattered Raman light is diffracted by a $2400 \mathrm{~g} / \mathrm{mm}$ grating and detected by a charged coupled device camera. The spectral distance between adjacent channels is $\sim 0.5$ $\mathrm{cm}^{-1}$. This setup enables the determination of Raman shift with an accuracy of $0.1 \mathrm{~cm}^{-1}$. The He-Cd plasma line at $854.7 \mathrm{~cm}^{-1}$ was used for the calibration of Raman spectra. It is important to note that the $\mathrm{SiO}_{2}$ layer is transparent to the UV laser which reaches the underlying Si substrate. This source of background must be subtracted in the analysis of the different spectra of the patterned nanostructures. The strain values are calculated from the measured wavenumber of Si-Si phonons using (13): 


$$
\omega_{S i-S i}^{\text {strained }}=\omega_{S i-S i}^{\text {bulk }}+\frac{\omega_{S i-S i}^{\text {bulk }}}{2}\left(q+\frac{S_{12}}{S_{11}+S_{12}} p\right) \times\left(\varepsilon_{x x}+\varepsilon_{y y}\right)
$$

where $\omega_{S i-S i}^{\text {bulk }}$ and $\omega_{S i-S i}^{\text {strained }}$ are Si-Si Raman shift frequency (in $\mathrm{cm}^{-1}$ ) of bulk and strained Si, respectively, $\varepsilon_{x x}$ and $\varepsilon_{y y}$ represent the strain in the two in-plane directions, $s_{11}$ and $s_{12}$ are the anisotropic elastic compliance tensor elements, $p$ and $q$ are the phonon deformation potentials. $p, q, s_{11}$, and $s_{12}$ for $\mathrm{Si}$ are given in Refs. 13 and 14. Figure 2 shows a typical map of the average biaxial strain of a SSOI substrate obtained by using UV micro-Raman analysis.

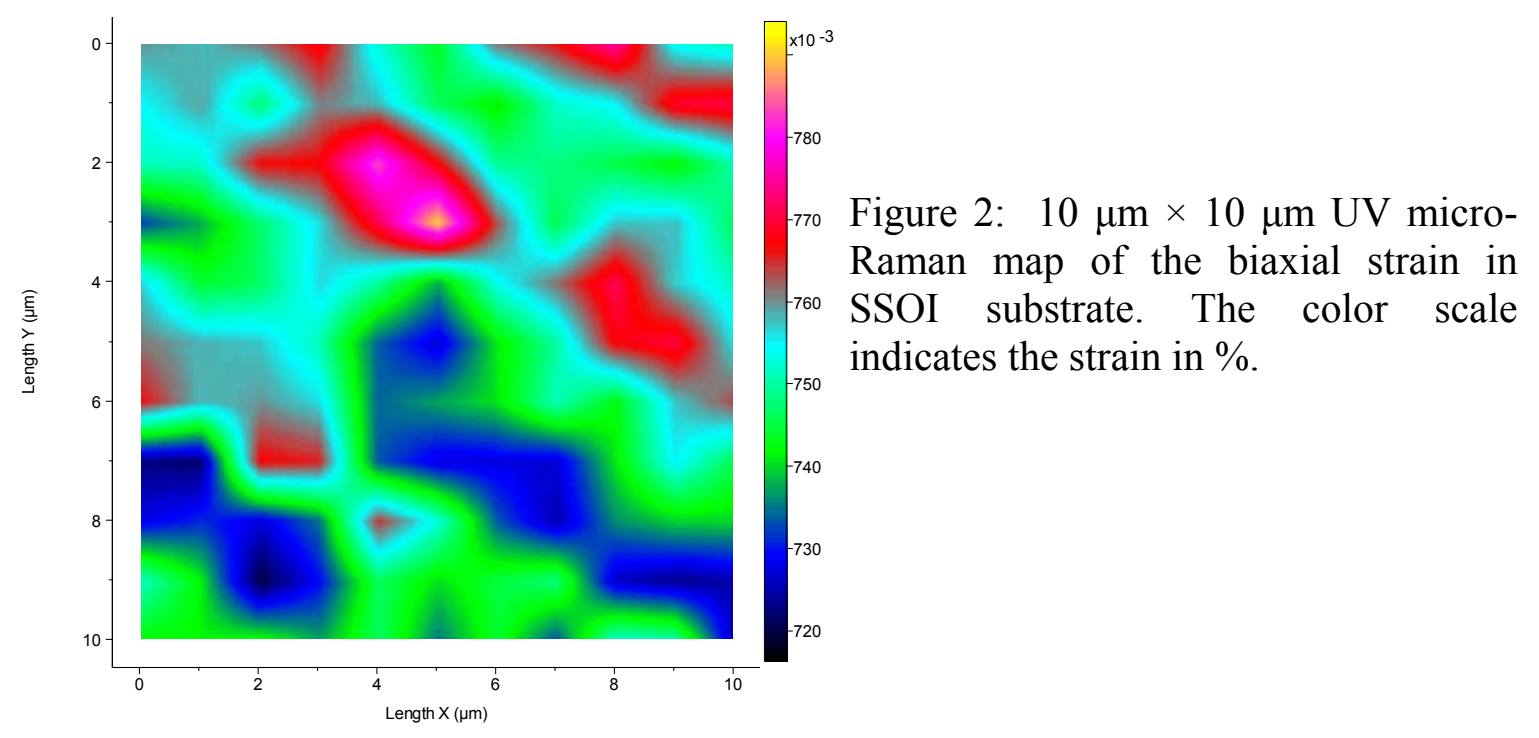

Besides UV Raman spectroscopy, HRXTEM is also used to quantify the strain in the strained layer. Assuming a constant spatial relationship between the intensity maxima in the HREM-image and the relative positions of the atomic columns in the specimen, strain can be mapped by the application of image processing techniques to the respective HREM-image. For that, several procedures have been developed in the last 15 years, which are mainly based on two different approaches: peak-finding and geometric phase analysis (15-21). By processing numerically the HRXTEM images it is possible to estimate the local shifts of intensity maxima with respect to the positions in a relaxed lattice. The peak-finding methods, operating in real space on the HRXTEM image, construct a lattice of points, which represent the actual atomic column positions, by a refined measurement of the location of intensity maxima. The comparison of that actual lattice with the point lattice associated with a non-distorted region gives the atomic displacements and, hence, the strain distribution. Figure 3 displays typical strain maps obtained by applying the peak-pairs analysis (PPA). Along the edges of the image black areas are observed, which are attributed to the correction of the geometrical distortions. The reference region of unstrained $\mathrm{Si}$ is shown on the right and the region of interest is marked by the white rectangle, from which the $\varepsilon_{\mathrm{xx}}$ values were finally calculated by integrating the corresponding profile in the strain map over a length of about $20 \mathrm{~nm}$ along the $<\underline{110}>$ direction. The estimated average strain $\varepsilon_{\mathrm{xx}}$ was found to be $\sim 1.2 \%$. We note that there is a certain speckling within the map, which might be caused by local crystal tilts, thickness variation and thin foil relaxation across the specimen. These effects generally limit the reliability of strain-mapping methods based on processing of HREM- 
images $(17,21,22)$. Nevertheless, the $\varepsilon_{\mathrm{xx}}$ component of the strain field in $\langle 110\rangle$ direction was calculated by the PPA method to amount about $1.2 \%$, which agrees well with the value obtained by Raman-spectroscopy. Expectedly, no strain is detected along $z$-axis.
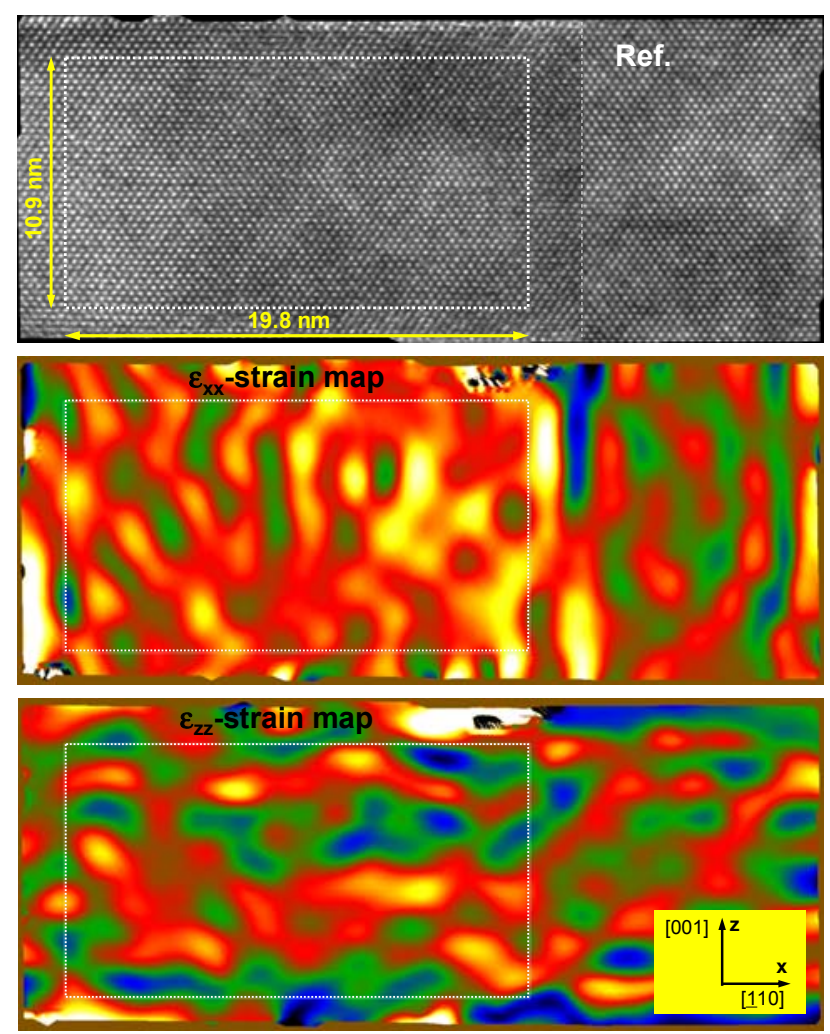

Figure 3. Strain maps of a SSOI substrate as obtained using PPA procedure. Top: HRXTEM images of SSOI (left) and bulk $\mathrm{Si}$ (right). Strain distribution along $x$ (middle) and $z$ (bottom) directions. Note that the substrate analyzed here is different from the one given in figure 2 .

\section{Nanoscale patterning of SSOI and strain evolution}

Data presented above demonstrate that the fabricated SSOI wafers are defect-free and uniformly biaxially tensile-strained. In the following we address the evolution of the strain upon nanoscale pattering. Extended $\left(400 \times 400 \mu \mathrm{m}^{2}\right)$ ordered arrays of rectangular pillars with a width in the range of 80 to $200 \mathrm{~nm}$ and a length of 200 or $400 \mathrm{~nm}$, separated by about $500 \mathrm{~nm}$, were patterned on a negative resist using electron-beam lithography. Reactive ion etching (RIE) was applied to transfer the pattern to the strained layer leading to ordered arrays of rectangular strained $\mathrm{Si}$ nanostructures directly on $\mathrm{SiO}_{2}$ (nanoSSOI). Figure 4 displays selected scanning electron microscopy micrographs of the arrays of nanoSSOI studied in this work. Note that outside the patterned region the strained $\mathrm{Si}$ layer was completely etched away. The patterned structures were characterized by UV micro-Raman. As mentioned above, Raman signal contains also the contribution of the underlying substrate. Therefore, the intensity of the intrinsic Si-Si Raman mode corresponding to a given nanostructure can be expressed as follow:

$$
I_{S i-S i}^{\text {Intrinsic }}=I_{S i-S i}^{\text {pattern }}-F_{\text {Norm }} \times I_{S i-S i}^{S i O 2 / S i}
$$

where $I_{S i-S i}^{\text {pattern }}$ and $I_{S i-S i}^{S i O 2 / S i}$ are Raman intensities collected from the patterned SSOI and the $\mathrm{SiO}_{2} / \mathrm{Si}$ (out of the pattern) regions, respectively. $F_{\text {Norm }}$ is the normalization factor 
which corresponds to the normalized inter-pillars area (i.e., $F_{\mathrm{Norm}} \times I_{\mathrm{Si}-\mathrm{Si} / \mathrm{Si}}$ presents the normalized background).
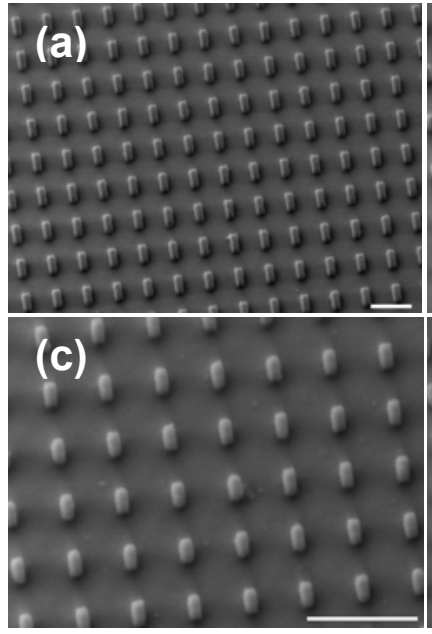
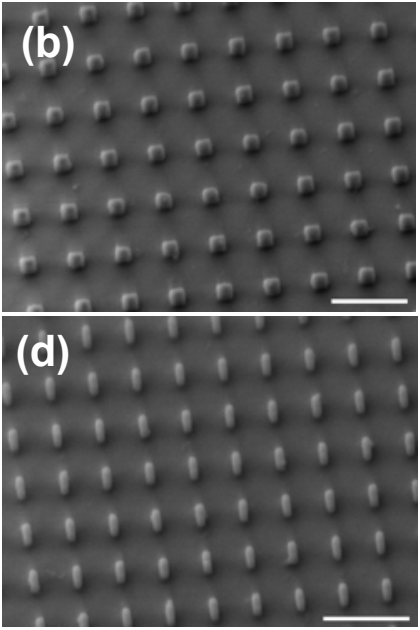

Figure 4. SEM images of the ordered arrays of some nanostructured SSOI investigated in this work. (a) $400 \mathrm{~nm} \times$ 200nm; (b) $200 \mathrm{~nm} \times 200 \mathrm{~nm}$; (c) 120 $\mathrm{nm} \times 200 \mathrm{~nm}$; and (d) $80 \mathrm{~nm} \times 200 \mathrm{~nm}$. The scale bar is $1 \mu \mathrm{m}$.

Figure 5 displays the Si-Si intrinsic Raman spectra of different nanoSSOI structures. The peak position is determined by a Voigt function fit and the sum of the in-plane strains is evaluated using Eq.1. We note that all the spectra are shifted up compared to the $\mathrm{Si}-\mathrm{Si}$ peak position of the strained film. These observations suggest that the patterning process induces a relaxation of the tensile strain. This shift is about $2.2 \mathrm{~cm}^{-1}$ for the largest structure $400 \mathrm{~nm} \times 200 \mathrm{~nm}$. The more the size shrinks the more the intrinsic Si-Si peak position moves closer to the bulk frequency indicating a more pronounced relaxation. The smallest structures $80 \mathrm{~nm} \times 200 \mathrm{~nm}$ and $80 \mathrm{~nm} \times 80 \mathrm{~nm}$ seem to be fully relaxed.

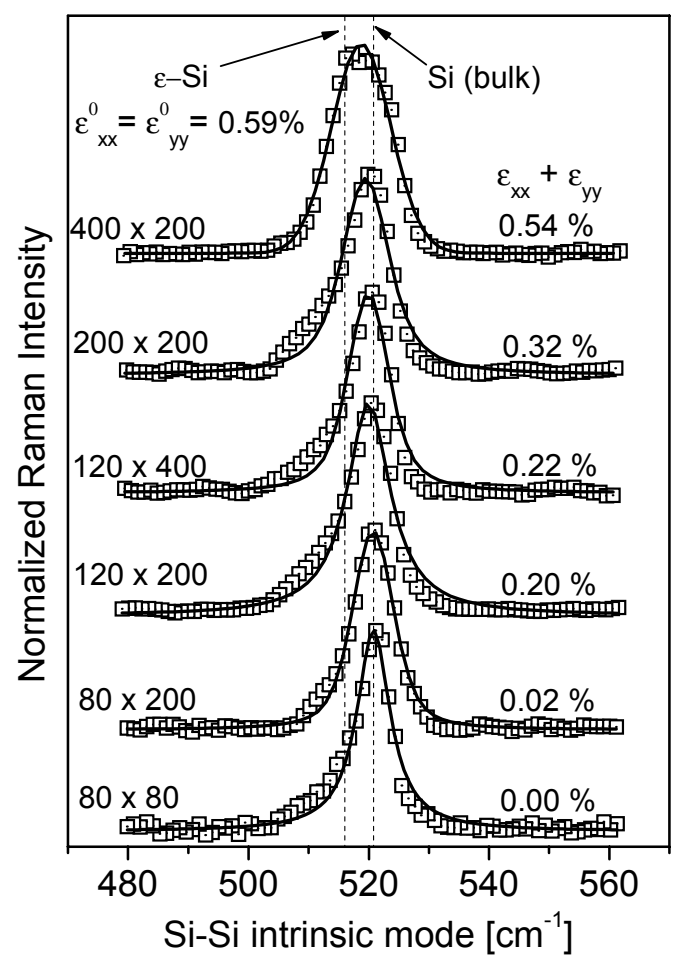

Figure 5: Si-Si intrinsic modes extracted for different dimensions. The corresponding sum of longitudinal and transverse in-plane strain is indicated. The open symbols present the experimental data and the solid lines the Voigt function fits. Dashed lines show the SiSi peak positions for both the initial SSOI substrate and the bulk Si. 
The origin of the observed relaxation resides in the formation of free surfaces during the pattering process. In general, this edge effect leads to the relaxation of the tensile strain following different mechanisms: 1- The edges of the strained nanostructure move inwards and drag the underlying substrate along with them (23); 2- The edges move inwards without any distortion of the substrate $(24,25)$; and 3- The edges bend up and the central region bends down, making it concave (13). For nanoscale-sized structures surface stress due to atomic rearrangement at the patterning-induced free surfaces can also affect the strain state. However, the contribution of the surface reconstruction can only be significant for dimensions in the order of a few nanometers (26). Therefore, it is reasonable to ignore the surface effects in the structures investigated in this work.

Due to their asymmetry, the residual strain in rectangular nanostructures is no more bisotropic (the strains in the two in-plane directions, $\varepsilon_{x x}$ and $\varepsilon_{y y}$, are different). Unfortunately, in backscattering geometry, Raman selection rules forbid the detection of the TO phonons. Therefore, the individual stress tensor elements can not be resolved. In order to gain more insights into the complexity of the relaxation phenomenon, we performed detailed three dimensional (3D) finite-element (FE) simulations using the program Ansys 11.0. Selected data of the 3D distribution of the simulated equivalent strain are shown in Fig. 6. We note that the edges and the surface undertake a strong contraction and the residual equivalent strain drops to values below $0.1 \%$. Expectedly, for the square nanostructure (Fig. 6 (a)) the relaxation process is symmetric along the two in-plane directions. A pronounced contraction was observed for the smallest structure 80 $\mathrm{nm} \times 80 \mathrm{~nm}$ (not shown) in agreement with the experimental data (Fig. 5) (10). It is worth pointing out that the strain becomes anisotropic in rectangular nanostructures. Independently of the size and shape, the lateral relaxation induces a vertical distortion of the lattice (initially $\varepsilon_{z z}=0$ ). Additionally, the simulations show that the relaxation induces a very strained region at the interface $\mathrm{Si} / \mathrm{SiO}_{2}$ (the gray zones in Fig. 6). Close examination of the cross section of the simulated 3D maps indicates that the edge of the interface $\mathrm{Si} / \mathrm{SiO}_{2}$ becomes highly strained upon nanopatterning. This indicates that the relaxation induces a distortion of the $\mathrm{SiO}_{2}$ layer as it is expected from the mechanisms 1 and 3 described above.

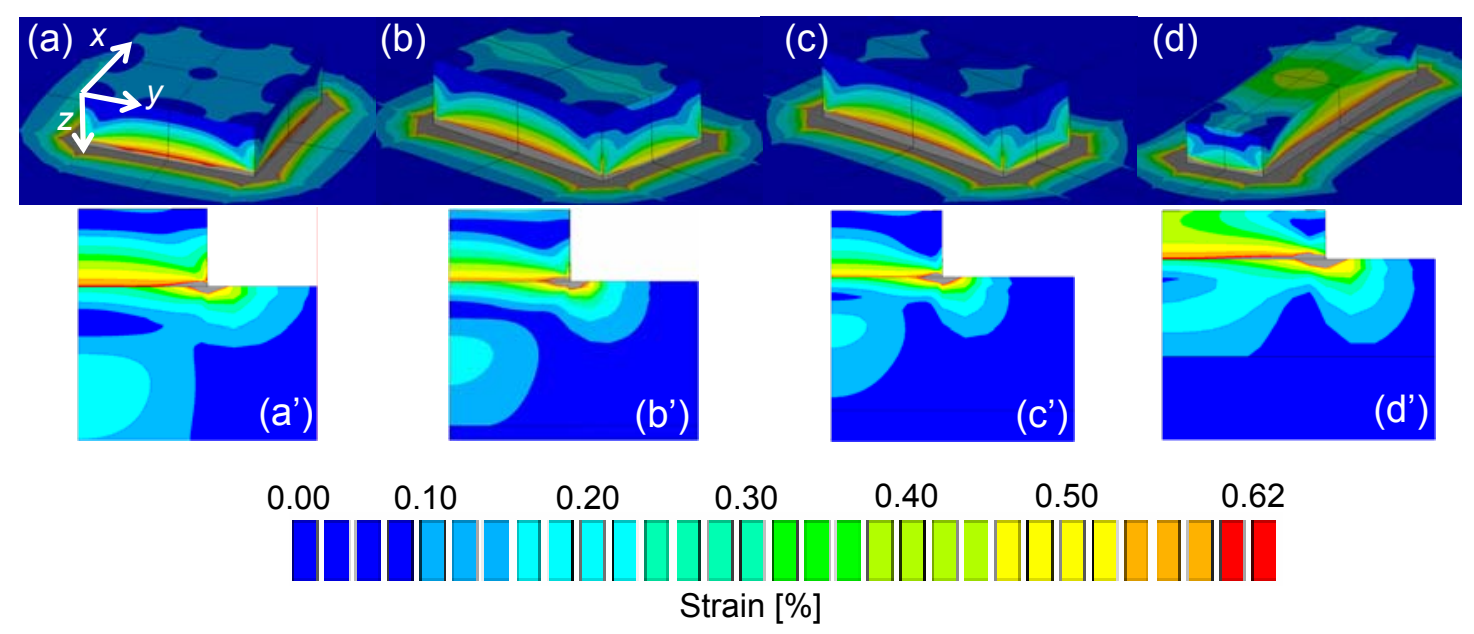

Figure 6: 3D FE simulations of the equivalent strain distribution within nanoSSOI with different dimensions: (a) $200 \mathrm{~nm} \times 200 \mathrm{~nm}$; (b) $120 \mathrm{~nm} \times 200 \mathrm{~nm}$; (c) $80 \mathrm{~nm} \times 200 \mathrm{~nm}$; and (d) $120 \mathrm{~nm} \times 400 \mathrm{~nm}$. (a'-d') are the corresponding cross section views. The highly strained regions at the interface of nanoSSOI and $\mathrm{SiO}_{2}$ are off scale $(\sim 1.4 \%)$. 
The observed anisotropy in the relaxation in rectangular structures is related to Poisson effect by which the contraction along the long dimension can induce an additional tensile strain along the short dimension (27). Hashemi et al. have exploited this anisotropic relaxation to fabricate uniaxial tensile strained $\mathrm{Si}$ nanowires $(w<<L)$ by patterning a biaxial tensile strain film (28). We anticipate that further manipulation of the strain in nanopatterned SSOI can be achieved by adjusting their thickness and a controlled manipulation of the mechanical properties of the underlying oxide by tuning its stoichiometry.

\section{Conclusion}

In conclusion, we described the strain mapping in sSOI substrates by using UV micro-Raman and PPA processing of HRXTEM data. We also studied the influence of the size on the strain evolution upon nanoscale patterning of $60 \mathrm{~nm}$-thick SSOI substrates. UV micro-Raman analysis demonstrated that the strain in patterned structures relaxes due to the formation of free surfaces. Under our experimental conditions, the biaxial strain is completely relieved in the patterned structures with a lateral dimension of $80 \mathrm{~nm}$. The evolution of the strain was also studied by 3D FE simulations. The simulated 3D maps demonstrate that the relaxation is pronounced at the edges and at the surface. Independently of the size, the simulations indicate that the $\mathrm{Si} / \mathrm{SiO}_{2}$ interface becomes highly strained and the patterned structures exhibit a out-of-plane distortion. The anisotropic relaxation in rectangular nanostructures was also discussed.

\section{Acknowledgments}

We are grateful to U. Doss and H. Blumtritt for their technical help. This work was supported by the German Federal Ministry of Education and Research in the framework of the DECISIF project (contract no. 13 N 9881).

\section{References}

1. I. Cayrefoureq et al., ECS Transactions 3, 399 (2006).

2. G. Taraschi et al., J. Electrochem. Soc. 151, G47, (2004).

3. L. J. Huang et al., Symp. VLSI Techn. Dig. 57, (2001).

4. S. E. Thompson, S. Suthram, Y. Sun, G. Sun, S. Parthasarathy, M. Chu, T. Nishida, IEDM Tech. Digest, 1 (2006).

5. M. Reiche, O. Moutanabir, C. Himcinschi, S. Christiansen, W. Erfurth, U. Gösele, S. Mantl, D. Buca, Q.T. Zhao,R. Loo, D. Nguyen, F. Muster, and M. Petzold, ECS Trans. 16, 311 (2008).

6. G. Abstreiter, H. Brugger, T. Wolf, H. Jorke, and H. J. Herzog, Phys. Rev. Lett. 54, 2441 (1985).

7. K. Kim, C.-T. Chuang, K. Rim, and R. Joshi, Sol. Stat. Elect. 48, 239 (2003).

8. S. J. Koester, K. Rim, J. O. Chu, P. M. Mooney, J. A. Ott, and M. A. Hargrove, Appl. Phys. Lett. 79, 2148 (2001).

9. C. Himcinschi, R. Singh, I. Radu, A. P. Milenin, W. Erfurth, M. Reiche, U. Gösele, S. H. Christiansen, F. Muster, and M. Petzold, Appl. Phys. Lett. 90, 021902 (2007). 
10. O. Moutanabbir, M. Reiche, W. Erfurth, F. Naumann, M. Patzold, and U. Gösele, Appl. Phys. Lett. (in press).

11. M. Reiche, C. Himcinschi, U. Gösele, S. H. Christiansen, S. Mantl, D. Buca, Q. T. Zhao, S. Feste, R. Loo, D. Nguyen, W. Buchholtz, A. Wei, M. Horstmann, D. Feijoo, and P. Storck, ECS Trans. 6, 339 (2007).

12. H.S.P. Wong, IBM Journal of Research and Development 46, No 2/3 (2002).

13. S. C. Jain, B. Dietrich, H. Richter, A. Atkinson, and A. H. Harker, Phys. Rev. B 52, 6247 (1995).

14. W. A. Brantley, J. Appl. Phys. 44, 534 (1973).

15. R. Bierwolf, H. Hohenstein, F. Philipp, O. Brandt, G.E. Crook, K. Ploog, Ultramicroscopy 49, 273 (1993).

16. A. Rosenauer, Transmission Electron Microscopy of Semiconductor Nanostructures An Analysis of Composition and Strain State, Springer Tracts in Modern Physics, Vol. 182, Springer-Verlag Berlin Heidelberg New York, 2003, ISBN 3-540-00414-9

17. P. Galindo, S. Kret, A.M. Sanchez, J.-Y. Laval,A. Yanez, J. Pizarro, E. Guerrero, T. Ben, S.I. Molina, Ultramicroscopy 107, 186 (2007).

18. D.L. Sales, J. Pizarro, P.L. Galindo, R. Garcia, G. Trevisi, P. Frigeri, L. Nasi, S. Franchi, S.I. Molina, Nanotechnology 18, 475503 (2007).

19. PPA-software, the GatanTM DigitalMicrograph plug-in for High-Resolution Peak Measurement and Strain Mapping Analysis, Version 1.01, HREM research Inc., http://www.hremresearch.com/

20. M.J. Hÿtch, E. Snoeck, R. Kilaas, Ultramicroscopy 74, 131 (1998) 131.

21. M.J. Hÿtch, T. Plamann, Ultramicroscopy 87, 199 (2001).

22. K. Du, F. Phillipp, Journal of Microscopy 221, 63 (2006).

23 S. M. Hu, J. Appl. Phys. 50, 4661 (1997).

24 S. Sakai, K. Kawasaki, and Wada, Jpn. J. Appl. Phys. 29, L853 (1990).

25. P. Van Mieghem, S. C. Jain, J. Nijs, and R. Van Overstraeten, J. Appl. Phys. 75, 666 (1994).

26. F. Liu, M. G. Lagally, and J. Zang, MRS Bull. 34, 190 (2009)

27. see for e.g., R. L. Peterson, K. D. Hobart, H. Yin, and J. C. Sturm, Proceedings of the 2004 IEEE International SOI Conference, 39 (2004).

28. P. Hashemi, M. Canonico, J. K. W. Yang, L. Gomez, K. K. Berggren, and J. L. Hoyt, ECS Transactions 16, 57 (2008). 